\title{
Traffic Forecasting and Planning of WiMAX under Multiple Priority Using Fuzzy Time Series Analysis
}

\author{
Ismail Bin Abdullah, Daw Abdulsalam Ali Daw, Kamaruzzaman Bin Seman \\ Faculty of Science and Technology, Universiti Sains Islam Malaysia (USIM), Bandar Baru Nilai, Malaysia \\ Email: isbah@usim.edu.my
}

Received December 2014

\begin{abstract}
Network traffic prediction plays a fundamental role in characterizing the network performance and it is of significant interests in many network applications, such as admission control or network management. Therefore, The main idea behind this work, is the development of a WIMAX Traffic Forecasting System for predicting traffic time series based on the daily and monthly traffic data recorded (TRD) with association of feed forward multi-layer perceptron (FFMLP). The quality of forecasting WIMAX Traffic obtained by comparing different configurations of the FFMLP that consist of investigating different FFMLP model architectures and different Learning Algorithms. The decision of changing the FFMLP architecture is essentially based on prediction results to obtain the FFMLP model for flow traffic prediction model. The different configurations were tested using daily and monthly real traffic data recorded at each of the two base stations (A and B) that belongs to a Libyan WiMAX Network. We evaluate our approach with statistical measurement and a good statistic measure of FMLP indicates the performance of selected neural network configuration. The developed system indicates promising results in which it successfully network traffic prediction through daily and monthly traffic data recorded (TRD) association with artificial neural network.
\end{abstract}

\section{Keywords}

Network Traffic, Wimax, Fuzzy Time Series, Forecasting

\section{Introduction}

Fuzzy systems are systems combining fuzzifier, fuzzy rule bases, fuzzy inference engine and defuzzifier (Wang, [1]). The systems have advantages that the developed models are characterized by linguistic interpretability and the generated rules can be understood, verified and extended. As a universal approximator, fuzzy systems have capability to model non stationary time series and give effect of data pre-processing on the forecast performance (Zhang, et al., [2] [3]; Zhang \& Qi, [4]). Studying on data pre-processing using soft computing method has been done. Popoola [4] has analyzed effect of data pre-processing on the forecast performance of subtractive cluster- 
ing fuzzy systems. Then, Popoola [5] has developed fuzzy model for time series using wavelet-based preprocessing method. Wang [1] and Tseng, et al. [6] applied fuzzy model to analyze financial time series data. Not all kinds of series data can be analyzed by conventional time series methods. Song \& Chissom [7] introduced fuzzy time series as a dynamic process with linguistic values as its observations. Techniques to model fuzzy time series data are based on fuzzy systems. Network traffic prediction plays a fundamental role in characterizing the network performance [8] and it is of significant interests in many network applications, such as admission control or network management. Therefore, The main idea behind this work, is the development of a WIMAX Traffic Forecasting System for predicting traffic time series based on the daily and monthly traffic data recorded (TRD) with association of feed forward multi-layer perceptron (FFMLP) [9]. The quality of forecasting WIMAX Traffic obtained by comparing different configurations of the FFMLP that consist of investigating different FFMLP model architectures and different Learning Algorithms [10].

The decision of changing the FFMLP architecture is essentially based on prediction results to obtain the FFMLP model for flow traffic prediction model. The different configurations was tested using daily and monthly real traffic data recorded at each of the two base stations (A and B) that belongs to a Libyan WiMAX Network. We evaluate our approach with statistical measurement and a good statistic measure of FMLP indicates the performance of selected neural network configuration [11]. The developed system indicates promising results in which it successfully network traffic prediction through daily and monthly traffic data recorded (TRD) association with artificial neural network.

\section{Fuzzy Time Series}

The fuzzy time series model proposed by Song and Chissom [7] consists of two major processes: 1) fuzzification and 2) the establishment of fuzzy relation relationships and forecasting. One study has pointed out that during the fuzzification process, different lengths of intervals will result in various forecasting results, and it has been proposed that the effective lengths of intervals be used [4]. The forecasting results that were based on the effective lengths of intervals were found to outperform those based on arbitrary ones.

Chen's method uses a simple operation, instead of complex matrix operations, in the establishment step of fuzzy relationships. The algorithm of Chen's method can be given as follows:

Step 1. Define the universe of discourse and intervals for rules abstraction. Based on the issue domain, the universe of discourse can be defined as: $\mathrm{U}=$ [starting, ending]. As the length of interval is determined $\mathrm{U}$ can be

Partitioned into several equally length intervals.

Step 2. Define fuzzy sets based on the universe of discourse and fuzzify the historical data.

Step 3. Fuzzify observed rules.

Step 4. Establish fuzzy logical relationships (FLRs) and group them based on the current states of the data of the fuzzy logical relationships.

Step 5. Forecast. Let $F(t-1)=A_{i}$

Case 1: If the fuzzy logical relationship of $A_{i}$ is empty; $A_{i} \rightarrow \emptyset$, then $F(t)$, forecast value, is equal to $A_{i}$.

Case 2: There is only one fuzzy logical relationship in the fuzzy logical relationship sequence. If $A_{i} \rightarrow A_{j}$, then $F(t)$, forecast value, is equal to $A_{j}$.

Case 3: If $A_{i} \rightarrow A_{j 1}, A_{j 2}, \ldots, A_{j k}$, then $F(t)$, forecast value, is equal to $A_{j 1}, A_{j 2}, \ldots, A_{j k}$

Step 6. Defuzzify. If the forecast of $F(t)$ is $A_{j 1}, A_{j 2}, \ldots, A_{j k}$, the defuzzified result is equal to the arithmetic average of the midpoints of $A_{j 1}, A_{j 2}, \ldots, A_{j k}$.

\section{Methodology}

Apart with some modifications Fuzzy Time Series (FTS) models have achieved successes in their own linear or nonlinear domains. Zhang [12] stated that since it is difficult to completely know the characteristics of the data in a real problem, hybrid methodology can be a good strategy for practical use. Liu [13] introduced a combination between decomposition method and FTS for forecasting trend and seasonal time series. In this paper, we proposed a combination method between differencing as data preprocessing method [14] and FTS models for forecasting trend and seasonal time series [15].

Case (1): WIMAX traffic of userA

Trying to estimate the WiMAX Traffic from userA using the daily, weekly and monthly recorded data in- 
cludes the maximum and the minimum number of online users only, so the Traffic from userA of daily data give as:

$$
T \text { Daily (A) } \quad f \text { UserA (X user (Max), X user(Min)) }
$$

Case (2): WIMAX traffic of userB

Trying to estimate the WiMAX Traffic from userA using the daily, weekly and monthly recorded data includes the maximum and the minimum number of online users only, so the Traffic from userB of daily data give as:

$$
T \text { Daily (B) } \quad f \text { UserB (X user (Max), X user(Min)) }
$$

Case (3): WIMAX traffic of userAB

Trying to estimate the WiMAX Traffic from userAB using the daily, weekly and monthly recorded data includes the maximum and the minimum number of online users only, so the Traffic from userB of daily data give as:

$$
T \text { Daily (AB) } \quad f \text { UserAB (X user (Max), X user(Min)) }
$$

where,

$T$ Daily(A, B, AB) represents the WiMAX traffic from userA, userB and userAB users (byte) respectively.

$X$ user (Max), $X$ user (Min) represent the maximum and the minimum number of online (Max-online) and (Min-online) respectively.

Three differencing process are used in this study, the original series become three new series:

$$
\begin{aligned}
& T A=\mathrm{F}(\mathrm{t})-\mathrm{F}(\mathrm{t}-1) \\
& T B=\mathrm{F}(\mathrm{t})-\mathrm{F}(\mathrm{t}-2) \\
& T A B=\mathrm{F}(\mathrm{t})-\mathrm{F}(\mathrm{t}-3)
\end{aligned}
$$

In summary, the proposed methodology of the FITS system consists of two steps. In the first step, a differencing is used to make stationary process. In the second step, a FTS model is developed to model the differencing data. In this second step, we apply four FTS models proposed by Chen [16], Yu [17], Cheng et al. [18], and Lee and Suhartono [19]. The proposed method exploits the unique feature and strength of FITS model in determining different patterns. Thus, it could be advantageous to model trend, seasonal, and nonlinear patterns separately by using different models and then combine the forecasts to improve the overall modeling and forecasting performance.

To validate the methodology of the FITS method for forecasting trend and seasonal time series data, a new algorithm is proposed as follows:

Step 1. Apply differencing to get the stationary process, $T A, T B$ and $T A B$

Step 2. Apply FTS method to model the differencing data and get the forecast component of the stationary process, $\mathrm{Y}_{\mathrm{A}}(\mathrm{t}), \mathrm{Y}_{\mathrm{B}}(\mathrm{t})$, and $\mathrm{Y}_{\mathrm{AB}}(\mathrm{t})$. In this step, four FTS methods proposed by Chen [16], Yu [17], Cheng et al. [18], and Lee and Suhartono [19] are applied to find the best forecasted values.

Step 3. Calculate the final forecast values at the same original data scale.

1. For UserA differencing data

$$
U A(\mathrm{t})=\mathrm{Y}_{\mathrm{A}}(\mathrm{t})+\mathrm{F}(\mathrm{t}-1)
$$

2. For UserB differencing data

$$
U B(\mathrm{t})=\mathrm{Y}_{\mathrm{B}}(\mathrm{t})+\mathrm{F}(\mathrm{t}-2)
$$

3. For both UserAB differencing data

$$
U A B(\mathrm{t})=\mathrm{Y}_{\mathrm{AB}}(\mathrm{t})+\mathrm{F}(\mathrm{t}-3)
$$

Now Mean Square Error can be calculated as below:

$$
\operatorname{MSE}=\left(\sum_{\mathrm{t}=1 \text { to }}\left[\{\mathrm{F}(\mathrm{t})-\mathrm{U}(\mathrm{t})\}^{2} / \mathrm{n}\right]\right)^{1 / 2}
$$

where $\mathrm{n}$ is the number of forecasts.

\section{Result and Discussions}

Weekly, monthly and daily data are taken in three category and intermediate of them as shown below: 

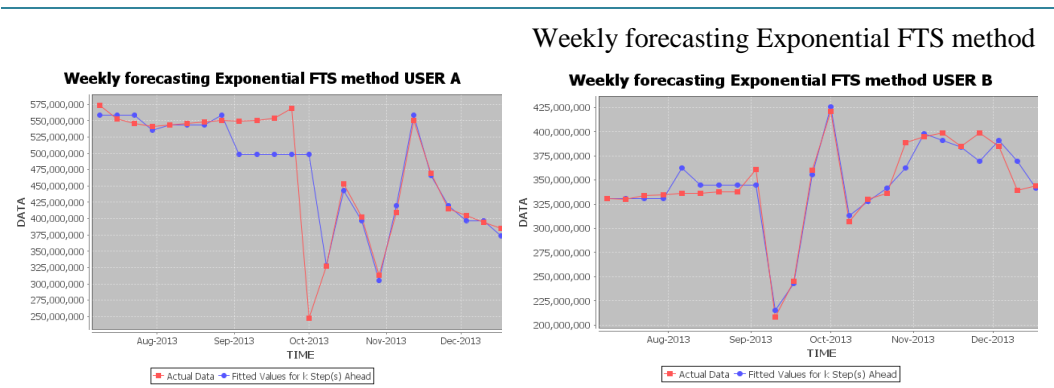

Monthly forecasting Exponential FTS method
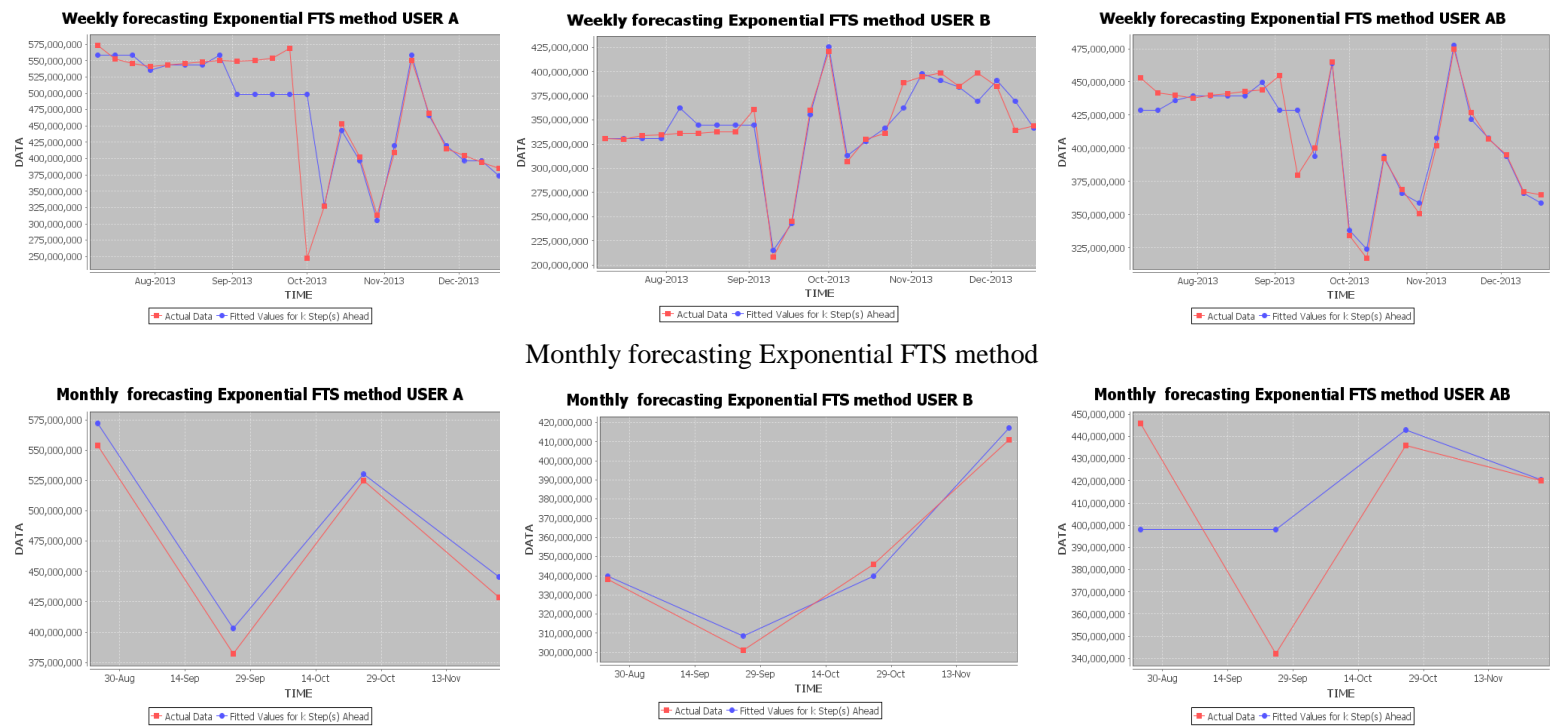

Daily forecasting Exponential FTS method
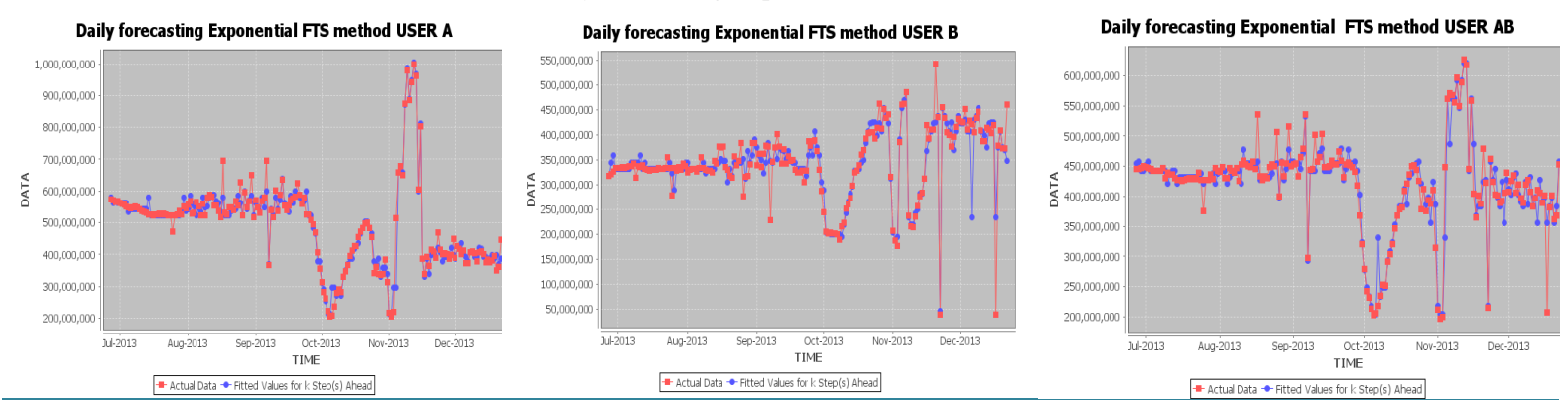

We have taken the traffic data with 51 in samples and 130 out samples for UserA, UserB and UserAB using FTS method is shown below:

Exponential FTS USER A: 2013m06d25

Daily forecasting Exponential FTS method USER A: Number of outsamples: 130; Number of insample: 51

D1: 100, d2: 100; Order 1,2

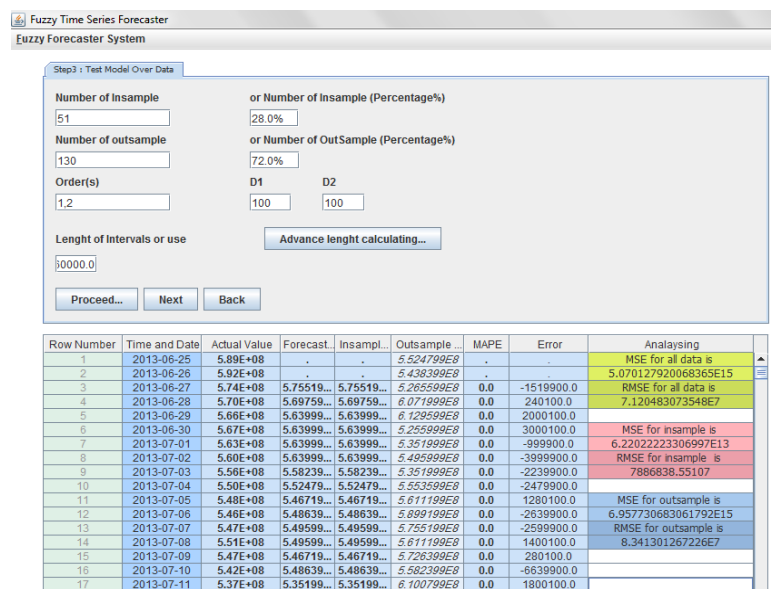

E:IFUZZY_forecasting_SYSTEM

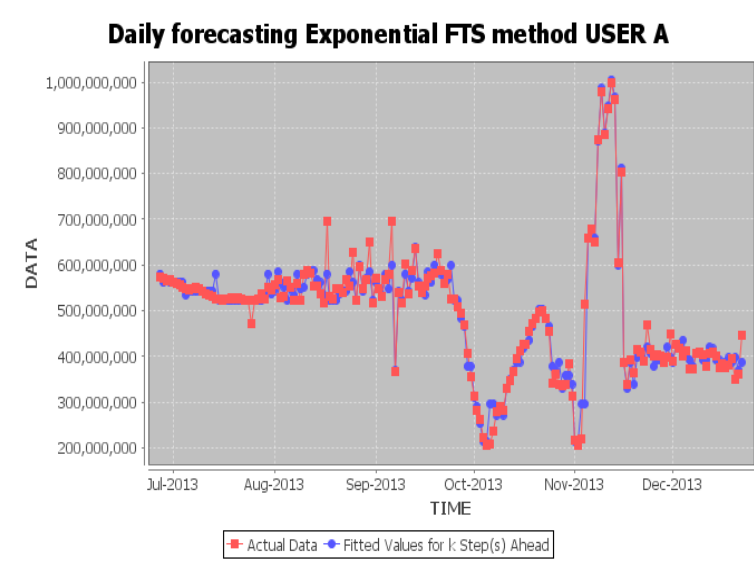

Exponential FTS USER B: 2013m06d25

Daily forecasting Exponential FTS method USER B: Number of outsamples: 130; Number of insample: 51 
D1: 100, d2: 100; Order 1,2

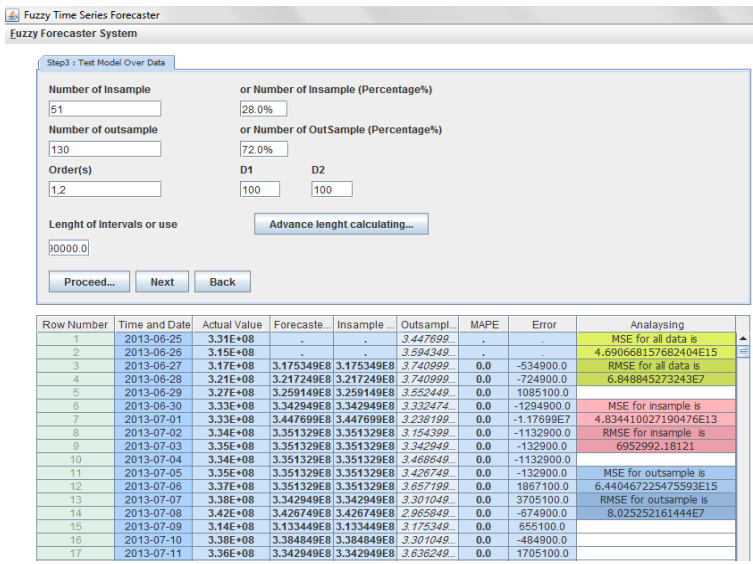

E:IFUZZY_forecasting_SYSTEM

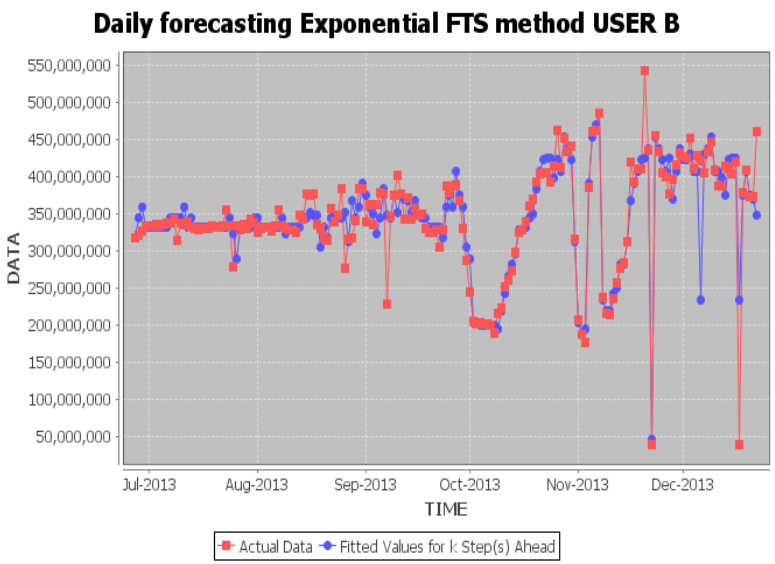

Exponential FTS USER AB: 2013m06d25

Daily forecasting Exponential FTS method USER AB; Number of outsamples: 130; Number of insample: 51

D1: 100, d2: 100; Order 1,2

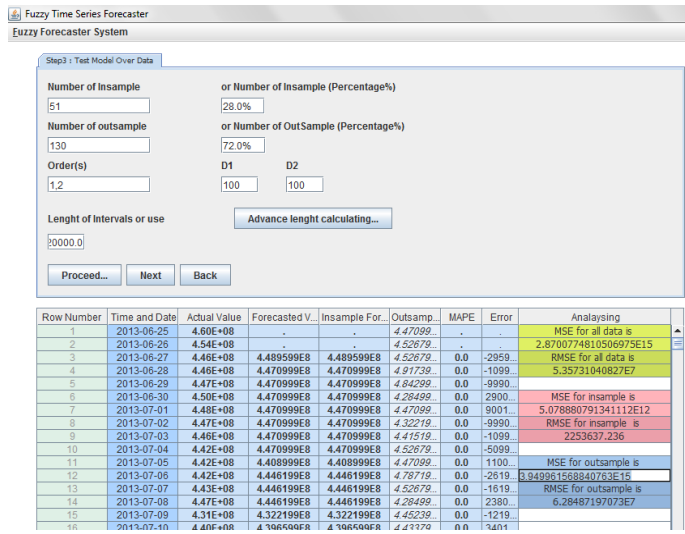

E:IFUZZY forecasting SYSTEM

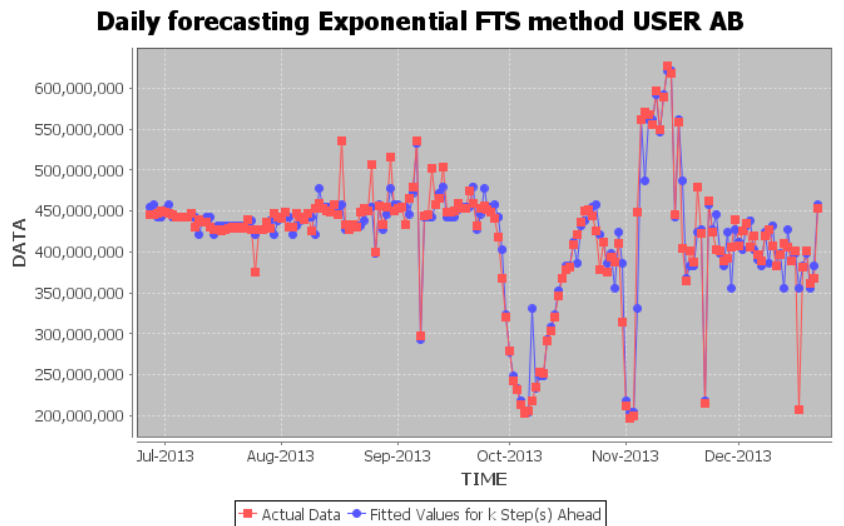

Similarly weekly and monthly data are taken for User A, B and AB
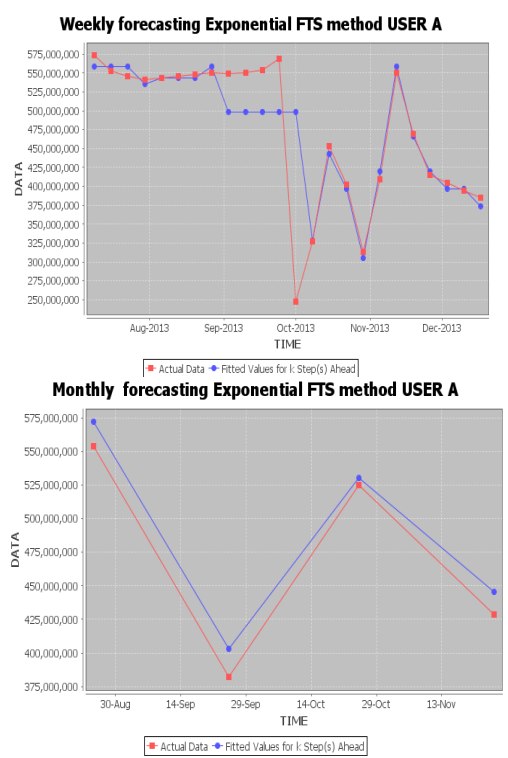

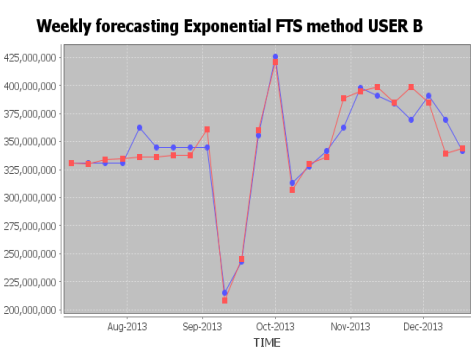

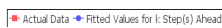

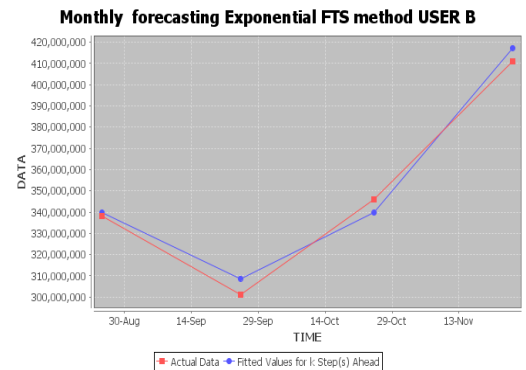

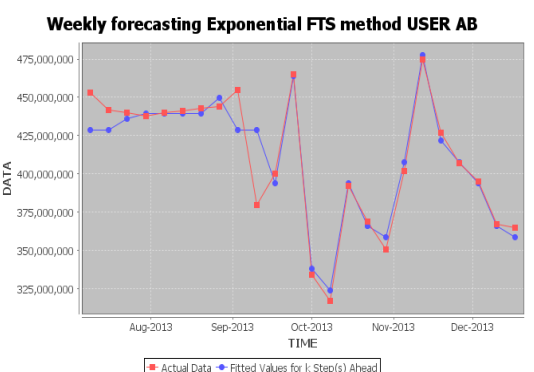

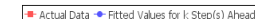

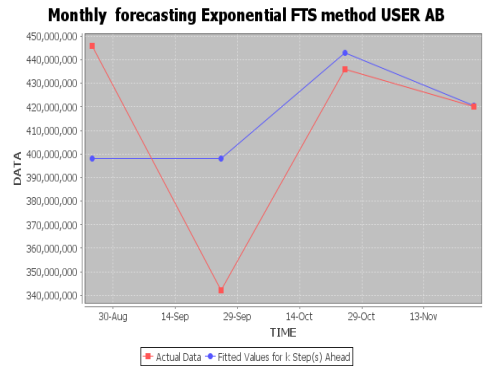


Daily fuzzy time series forecasting results

\begin{tabular}{|c|c|c|c|c|}
\hline & & USER A & USER B & USER AB \\
\hline \multirow{5}{*}{$\begin{array}{l}\text { Exponential FTS } \\
\text { weekly }\end{array}$} & Mse all & 8.117465852396059E15 & 2.924431681416891E15 & $1.994923193333558 \mathrm{E} 15$ \\
\hline & Mse-in & 3.12496250225E11 & 1.40000000225E11 & 3.89989500225E11 \\
\hline & Mser-o & $9.740896523625224 \mathrm{E} 15$ & 3.509290017700224E15 & 2.3938298341002245E15 \\
\hline & 1. intr & $\begin{array}{l}5500000.0 \\
2.31 \mathrm{E} 7\end{array}$ & $\begin{array}{c}1200000.0 \\
1.406 \mathrm{E} 7\end{array}$ & $\begin{array}{c}2400000.0 \\
1.398 \mathrm{E} 7\end{array}$ \\
\hline & MSE fitted v & 3.241700322458558E15 & 1.64951556125225E14 & $1.7434240980855834 \mathrm{E} 14$ \\
\hline \multirow{4}{*}{$\begin{array}{l}\text { Exponential FTS } \\
\text { monthly }\end{array}$} & Mse all & 8.122874861000004E15 & 2.367500078000004E15 & 3.226031221000004E15 \\
\hline & Mse-in & 2.49998000004E11 & 4.0 & 6.2501000004E10 \\
\hline & Mser-o & $1.0830416482000004 \mathrm{E} 16$ & 3.1566667706666705E15 & 4.3013541276666705E15 \\
\hline & 1. intr & $\begin{array}{c}3000000.0 \\
4.23 \mathrm{E} 7\end{array}$ & $\begin{array}{c}2000000.0 \\
1.55 \mathrm{E} 7\end{array}$ & $\begin{array}{c}2500000.0 \\
2.24 \mathrm{E} 7\end{array}$ \\
\hline \multirow{6}{*}{$\begin{array}{c}\text { Exponential FTS } \\
\text { Daily }\end{array}$} & MSE fitted v & 2.70037439000004E14 & $3.5312490500004 \mathrm{E} 13$ & 1.371599984800004E15 \\
\hline & Mse all & 5.070127920068365E15 & 4.690668157682404E15 & $2.8700774810506975 \mathrm{E} 15$ \\
\hline & Mse-in & 6.22022223306997E13 & 4.834410027190476E13 & 5.078880791341112E12 \\
\hline & Mser-o & $6.957730683061792 \mathrm{E} 15$ & 6.440467225475593E15 & 3.949961568840763E15 \\
\hline & 1. intr & $\begin{array}{c}5760000.0 \\
1.9322222 \mathrm{E} 7\end{array}$ & $\begin{array}{c}4190000.0 \\
1.5641667 \mathrm{E} 7\end{array}$ & $\begin{array}{c}3720000.0 \\
1.4936111 \mathrm{E} 7\end{array}$ \\
\hline & MSE fitted v & 8.423388576076724E14 & 9.384482805563819E14 & 6.57489415119946E14 \\
\hline
\end{tabular}

Mse all: mean square error for all; Mse-in: mean square error for insamples; Mse-o: mean square error for outsamples; l. intr: length of intervals.

The results shows that in case of Weekly data, overall MSE is least for Use rAB, insamples MSE is least for User B, out samples MSE is least for User AB, intermediate MSE is least for User B and MSE fiited v is least for User B.

In case of Monthly data, overall MSE is least for User B, in samples MSE is least for User A, outsamples MSE is least for User B, intermediate MSE is least for User B and MSE fited v is least for User AB.

In case of Daily data, overall MSE is least for Use $\mathrm{AB}$, in samples MSE is least for User B, outsamples MSE is least for User $\mathrm{AB}$, intermediate MSE is least for User AB and MSE fiited $v$ is least for User AB.

\section{Conclusion}

In this paper we have used FTS method for forecasting of WIMAX data traffic inbound and outbound which leads to seeking for accuracy in the term of Mean Square Error (MSE). The modeling uses classical FTS to the weighted FTS and finally the differencing provides the accurate results near to actual ones.

\section{Acknowledgements}

The authors thank LibyaMax network (WiMAX technology) motorized by Libya Telecom and Technology forproviding us the WiMAX traffic data and for helpful discussions around WiMAX network. We also thank staff of Faculty of Science and Technology, University Sains Islam Malaysia for their help and support.

\section{References}

[1] Wang, L.X. (1997) A Course in Fuzzy Systems and Control. Prentice-Hall, Inc., New Jersey.

[2] Zhang, G., Patuwo, B.E. and Hu, M.Y. (1998) Forecasting with Artificial Neural Networks: The State of the Art. International Journal of Forecasting, 14, 35-62. http://dx.doi.org/10.1016/S0169-2070(97)00044-7

[3] Zhang, B.-L., Coggins, R., Jabri, M.A., Dersch, D. and Flower, B. (2001) Multiresolution Forecasting for Future Trad- 
ing Using Wavelet Decomposition. IEEE Transactions on Neural Networks, 12, 765-775. http://dx.doi.org/10.1109/72.935090

[4] Zhang, G.P. and Qi, M. (2005) Neural Network Forecasting for Seasonal and Trend Time Series. European Journal of Operation Research, 160, 501-514. http://dx.doi.org/10.1016/j.ejor.2003.08.037

[5] Popoola, A.O. (2007) Fuzzy-Wavelet Method for Time Series Analysis, Dissertation. Department of Computing, School of Electronics and Physical Sciences, University of Surrey, Guildford, UK.

[6] Tseng, F.-M., Tseng, G.-H., Yu, H.-C. and Yuan, B.J.C. (2001) Fuzzy ARIMA Model for Forecasting The Foreign Exchange Market. Fuzzy Sets and Systems, 118, 9-19. http://dx.doi.org/10.1016/S0165-0114(98)00286-3

[7] Song, Q. and Chissom, B.S. (1993) Forecasting Enrollments with Fuzzy Time series Part I. Fuzzy Sets and Systems, 54, 1-9. http://dx.doi.org/10.1016/0165-0114(93)90355-L

[8] Mitra, A. and Mitra, S. (2006) Modeling Exchange Rates Using Wavelet Decomposed Genetic Neural Networks. Statistical Methodology, 3, 103-124. http://dx.doi.org/10.1016/j.stamet.2005.07.002

[9] Daw, D.A.A. and Seman, K.B. (2013) Gateway to Wimax Profiling Services in Libya. International Journal of Engineering Research and Development, 7, 63-68.

[10] Ion Railean, D., Stolojescu, C., Moga, S. and Lenca, P. (2010) WIMAX Traffic Forecasting based on Neural Networks in Wavelet Domain. 2010 Fourth International Conference on Ion Research Challenges in Information Science (RCIS), 443-452.

[11] Firoiu, I. and Stolojescu, C. and Isar, A. (2009) Forecasting of WiMAX BS Traffic: Observations and Initial Models, Alcatel Lucent Technical Report, January 2009.

[12] Zhang, G.P. (2003) Time Series Forecasting Using a Hybrid ARIMA and Neural Network Model. Neurocomputing, 50, 159-175. http://dx.doi.org/10.1016/S0925-2312(01)00702-0

[13] Liu, H.T. (2009) An Integrated Fuzzy Time Series Forecasting System. Expert Systems with Applications, 36, 1004510053. http://dx.doi.org/10.1016/j.eswa.2009.01.024

[14] Yang, Q. and Xindong, W. (2006) 10 Challenging Problems in Data Mining Research. International Journal of Information Technology and Decision Making, 5, 597-604. http://dx.doi.org/10.1142/S0219622006002258

[15] Ibarra-Berastegi, G., Elias, A., Arias, R. and Barona, A. (2007) Artificial Neural Networks vs Linear Regression in a Fluid Mechanics and Chemical Modelling Problem: Elimination of Hydrogen Sulphide in a Lab-Scale Biofilter. IEEE/ACS International Conference on Computer Systems and Applications, 584-587.

[16] Chen, S.M. (1996) Forecasting Enrollments Based on Fuzzy Time Series. Fuzzy Sets and Systems, 81, 311-319. http://dx.doi.org/10.1016/0165-0114(95)00220-0

[17] Yu, H.K. (2005) Weighted Fuzzy Time-Series Models for TAIEX Forecasting. Physica A: Statistical Mechanics and its Applications, 349, 609-624. http://dx.doi.org/10.1016/j.physa.2004.11.006

[18] Cheng, C.H., Chen, T.L., Teoh, H.J. and Chiang, C.H. (2008) Fuzzy Time Series Based on Adaptive Expectation Model for TAIEX Forecasting. Expert Systems with Applications, 34, 1126-1132. http://dx.doi.org/10.1016/j.eswa.2006.12.021

[19] Lee, M.H. and Suhartono (2010) A Novel Weighted Fuzzy Time Series Model for Forecasting Seasonal Data. Proceeding 2nd International Conference on Mathematical Sciences, Kuala Lumpur, Malaysia, 332-340. 